\title{
Pure laparoscopic versus open right hepatectomy in living liver donors: bench-surgery time and graft weight discrepancy
}

\author{
Kwangpyo Hong ${ }^{1}$, Suk Kyun Hong ${ }^{2}$, Eui Soo Han², Sanggyun Suh², Su young Hong ${ }^{2}$, Jeong-Moo Lee², YoungRok Choi ${ }^{2}$, \\ Nam-Joon $\mathrm{Yi}^{2}$, Kwang-Woong Lee ${ }^{2}$, Kyung-Suk Suh ${ }^{2}$

\footnotetext{
${ }^{2}$ Department of Surgery-Hepatobiliary, Seoul National University College of Medicine, Seoul, Korea
} \\ ${ }^{1}$ Department of Surgery-Hepatobiliary, Eulji University Hospital, Daejeon, Korea
}

Background: Recently, there have been several reports on pure laparoscopic donor right hepatectomy (PLDRH), but the affect of pure laparoscopy on bench-surgery has not been evaluated. This study aimed to compare bench-surgery time and the graft weight discrepancy between estimated- and actual weight of the liver graft in PLDRH in comparison with those of conventional donor right hepatectomy $(\mathrm{CDRH})$.

Methods: Prospectively collected medical records of 758 live liver donors between January 2012 and December 2019 were retrospectively reviewed. We divided it into two groups: between January 2012 and September 2015 when CDRH was performed exclusively and between March 2016 and December 2016 when PLDRH was standardized. We excluded all other type of graft donor hepatectomy, laparoscopic assisted donor hepatectomy, and no data recorded cases.

Results: Two hundred sixty-seven donors were included in PLDRH period and were compared with 247 donors who underwent in a period during which CDRH was performed exclusively. Similar proportion of graft vasculature variations were observed in the two groups. The mean bench-surgery time was longer in the PLDRH group than the CDRH group $(49.3 \pm 19.9$ vs. $39.5 \pm 17.5$ minutes; $P<0.001)$. The correlation of actual graft weight ( $A G W)$ and estimated graft volume (EGV) were significantly linear in two groups $(R=0.76, P<0.001$ and $R=0.80, P<0.001)$, but there was relatively higher linear correlation in PLDRH group.

Conclusions: The bench-surgery time takes longer in the PLDRH group regardless of reconstruction of vasculature or not. The correlation between EGV and AGW was more linear in PLDRH group.

Corresponding author: Suk Kyun Hong

E-mail: nobel1210@naver.com 